\title{
História de vida de adolescentes autistas: contribuições para a Fonoaudiologia e a Pediatria
}

\author{
Life stories of autistic adolescents: contributions to Speech-Language Therapy and Pediatrics
}

Maria Fernanda Bagarollo', Ivone Panhoca

\section{RESUMO}

Objetivo: Analisar os processos dialógicos de seis adolescentes autistas, durante terapia fonoaudiológica a respeito de suas histórias de vida, buscando subsídios para o pediatra e o fonoaudiólogo trabalharem com a família, a fim de impulsionar o desenvolvimento desses sujeitos.

Métodos: A coleta de dados foi realizada a partir da vídeogravação de duas sessões fonoaudiológicas com seis sujeitos diagnosticados como transtorno global do desenvolvimento, sendo três com transtorno autista e três com Asperger. As gravações foram transcritas e os dados, separados em eixos temáticos. As análises foram respaldadas na perspectiva qualitativa de pesquisa, sob a ótica da teoria histórico-cultural e o paradigma indiciário.

Resultados: Obtiveram-se as seguintes categorias a partir da coleta dos dados: lazer, escolaridade, grupo social, fatos da infância, vivências da adolescência e interesses. Tais categorias elucidaram as experiências vivenciadas pelos sujeitos e o modo como eles significam o mundo e são significados pelos outros, permitindo elencar dificuldades vivenciadas por esses sujeitos e seus familiares.

Conclusões: Os apontamentos dos sujeitos mostram a necessidade de orientações contínuas sobre os aspectos destacados nas categorias, desde o momento da descoberta do diagnóstico, proporcionando ao indivíduo uma participação social e um desenvolvimento linguístico-cognitivo mais efetivo. O pediatra e o fonoaudiólogo são os profissionais

Instituição: Programa de Pós-Graduação em Saúde da Criança e do Adolescente da Faculdade de Ciências Médicas da Universidade Estadual de Campinas (Unicamp), Campinas, SP, Brasil

1Doutora em Saúde da Criança e do Adolescente pela Faculdade de Medicina da Unicamp; Professora Colaboradora da Universidade Estadual do Centro-Oeste (Unicentro), Guarapuava, PR, Brasil

${ }^{2}$ Doutora pelo Instituto de Estudos da Linguagem da Unicamp; Professora Convidada do Programa de Pós-Graduação em Saúde da Criança e do Adolescente da Faculdade de Ciência Médicas da Unicamp, Campinas, SP, Brasil indicados para tais orientações, uma vez que são os primeiros a atender as crianças autistas e os que participam diretamente do momento da descoberta do diagnóstico, acompanhando os pacientes até a adolescência.

Palavras-chave: transtornos globais do desenvolvimento infantil; linguagem; grupo social; adolescente.

\section{ABSTRACT}

Objective: To analyze the dialogic processes of six autistic adolescents during speech-language therapy about their life stories, searching for support for pediatricians and speechlanguage therapists to work with their families in order to improve their development.

Methods: Data collection was carried out from video recordings of two speech-language therapy sessions with six subjects diagnosed with global development delay (three with autistic disorder and three with Asperger's disorder). The recordings were transcribed and data were separated into five themes. The analyses were based on qualitative research approach, in the context of the historical-cultural theory and the semiotic-indiciary paradigm.

Results: The following categories emerged from data collection: leisure, school level, social group, childhood facts, adolescence experiences and interests. Such categories reflected the experiences lived by the subjects and the way they signify the world and are signified by others. These

Endereço para correspondência:

Maria Fernanda Bagarollo

Rua Ângelo Orlando, 65 - Vila Medon

CEP 13465-180 - Americana/SP

E-mail: maria.fer@uol.com.br

Fonte financiadora: Coordenação de Aperfeiçoamento de Pessoal de Nível Superior/Bolsa Demanda Social.

Conflito de interesse: nada a declarar

Recebido em: 7/12/2009

Aprovado em: 8/6/2010 
categories allowed us to list the difficulties experienced by these subjects and their families.

Conclusions: The patients' indications reveal the need for continuous guidance about the aspects outlined in the categories, from the very moment that diagnosis is received, allowing the individual to have a more effective social participation and linguistic-cognitive development. The pediatrician and the speech-language therapist are the professionals to provide such guidance, once they are the first to work with autistic children and to actively participate of their lives, from the moment of diagnosis until adolescence.

Key-words: child development disorders, pervasive; language; social group; adolescent.

\section{Introdução}

O autismo é um distúrbio do desenvolvimento que ocasiona dificuldades nas interações do sujeito com as pessoas e com o mundo, alterações no funcionamento linguístico, comportamentos estereotipados, auto e heteroagressividade, distúrbios alimentares e do sono ${ }^{(1,2)}$. O quadro autístico configura-se como um espectro de sintomas que pode variar desde uma forma intensa de manifestação, sendo classificado como transtorno autista (299.00) ou, de maneira amena, como transtorno de Asperger (299.80) ${ }^{(3)}$. Atualmente, a nomenclatura utilizada para o autismo é transtorno global do desenvolvimento (TGD) $)^{(3)}$, porém aqui se utiliza também o termo autismo. Os dados epidemiológicos mostram que existem cerca de cinco sujeitos com o diagnóstico de TGD para cada 10 mil nascimentos ${ }^{(1)}$. Os critérios diagnósticos apontam que a patologia manifesta-se até a criança completar três anos e prolonga-se por toda a vida ${ }^{(3)}$.

Para além das alterações inerentes à patologia, estudos mostram que a vivência familiar e social desses indivíduos fica comprometida, na medida em que gera estresse familiar e dificuldades no modo de agir ${ }^{(4-9)}$. Tais dificuldades decorrem do fato de a organização da sociedade ser voltada para os padrões normais ${ }^{(10)}$, não oferecendo recursos suficientes e oportunidades para os sujeitos com o desenvolvimento atrelado a alguma deficiência e seus familiares. Todas as dificuldades enfrentadas pela família e pelo grupo social na convivência com os sujeitos com TGD iniciam-se com o aparecimento dos sintomas e agravam-se com o diagnóstico, podendo estender-se por toda vida.

Considerando-se a família o primeiro grupo social da criança - na qual lhe são proporcionadas aprendizagens e desenvolvimento, da qual ela vai se tornando participativa e pela qual é inserida nos demais grupos sociais, como a escola, a igreja, as atividades de lazer etc. - tais dificuldades podem ter consequências negativas para o desenvolvimento do sujeito autista, agravando mais o quadro patológico ${ }^{(10,11)}$.

A fase da descoberta do TGD é normalmente acompanhada por pediatras, na medida em que é uma especialidade médica que se dedica ao cuidado da criança e do adolescente em todos os níveis de complexidade ${ }^{(12)}$. Além disso, por ser o primeiro médico da criança, o pediatra passa a ser a referência para a família nas orientações sobre os cuidados e formas de tratamento que se deve ter em casa.

As alterações de linguagem costumam ser os primeiros sintomas a despertarem a preocupação da família e do pediatra, sendo o encaminhamento terapêutico inicial ${ }^{(13,14)}$ para a Fonoaudiologia e, posteriormente, para Psiquiatria e Neurologia. Desta maneira, o pediatra e o fonoaudiólogo passam a acompanhar o processo de desconfiança e diagnóstico do quadro de autismo, sendo relevante o conhecimento não só dos sintomas iniciais, mas de todas as dificuldades vivenciadas no decorrer da vida, a fim de orientar e direcionar a família quanto aos caminhos mais efetivos para o desenvolvimento de tais sujeitos.

Considerando-se o exposto acima, o objetivo deste trabalho foi observar processos dialógicos de sujeitos com diagnóstico de TGD a respeito de suas histórias de vida, buscando subsídios para o pediatra e o fonoaudiólogo orientarem e impulsionarem o desenvolvimento social, linguístico e cognitivo desses pacientes.

\section{Método}

Este projeto de pesquisa foi aprovado pelo Comitê de Ética em Pesquisa da Faculdade de Ciências Médicas da Universidade Estadual de Campinas (Unicamp). O termo de consentimento livre e esclarecido foi assinado pelos pais de todos os sujeitos participantes. Para as análises foi utilizado o método qualitativo, que se preocupa com a busca dos indícios, explicação e interpretação dos produtos humanos. Esse tipo de pesquisa considera o ser humano como parte de um processo filogenético, ontogenético e micrognético, sendo histórico, subjetivo e não quantificável. Essa perspectiva é respaldada teoricamente pela análise microgenética ${ }^{(15)}$ e pelo paradigma indiciário ${ }^{(16)}$.

Tal perspectiva metodológica considera que os dados de um sujeito ou de um grupo de sujeitos representam não apenas as questões individuais, mas também as concepções 
circulantes no grupo social ${ }^{(17)}$. Nessa perspectiva teórica, há preocupação menor com a generalização e maior com o aprofundamento e abrangência da compreenssão do objeto de estudo. Para respaldar tal afirmação, são tomadas as seguintes reflexões de Minayo ${ }^{(18)}$ : “A partir dos indivíduos, dos grupos ou das classes, a totalidade fundamental se expressa no perene conúbio entre mente e corpo, matéria e espírito; os sujeitos individuais projetam sua visão da sociedade e da natureza, a historicidade das relações e condições de produção inscritas no seu corpo, seu espaço hodológico, sua temporalidade social, seus infinitos culturais, seus fetiches, seus fantasmas e seus anseios de transcendência. Portanto, a pesquisa qualitativa reconhece o sujeito como autor, sob condições dadas, capaz de 'retratar' e 'refratar' a realidade" (p. 252). Nos termos acima citados, os dizeres dos adolescentes autistas trazem em seus conteúdos suas formas de pensar e entender a vida, o modo como a sociedade os significa e trata seus sentimentos.

Para escolha dos indivíduos que comporão uma dada amostra, a mesma autora aponta a necessidade de selecionar grupos sociais que detenham atributos que o pesquisador pretende investigar. A escolha dos sujeitos deve privilegiar a diversidade, a fim de permitir observar diferenças e semelhanças. O número de sujeitos deve atender ao critério de saturação, quando as informações são reincidentes nos da$\operatorname{dos}^{(18)}$. Na pesquisa qualitativa, não há a preocupação com o tratamento estatístico dos dados; no entanto, é fundamental a transparência ao se definir o perfil da população a ser estudada e o número dos sujeitos participantes.

Sobre o perfil da população deste estudo, é possível dizer que todos os sujeitos têm diagnóstico de TGD, residem na mesma cidade no interior do Estado de São Paulo (200 mil habitantes), são adolescentes entre 13 e 18 anos, moram com suas famílias, estão matriculados em escola regular ou especial (dependendo do grupo incluído para a pesquisa) e pertencem à classe média.

Quanto ao número de pacientes estudados, inicialmente foram levantados dados sobre o número de sujeitos cadastrados em serviços de saúde e educação da cidade em questão, encontrando-se 44 indivíduos com diagnóstico de TGD. Desses, 14 atendiam aos critérios desta pesquisa. Para a primeira etapa da coleta de dados, foram selecionados dez sujeitos, sendo que cinco estudavam em uma escola regular e enquadravam-se na definição de transtorno de Asperger ${ }^{(3)}$ e cinco estudavam em uma escola especial e enquadravam-se na definição de transtorno autista ${ }^{(3)}$. Iniciadas as entrevistas, observou-se que, após entrevistar três sujeitos de cada grupo, os dados se repetiam, já que os discursos de todos giravam em torno de questões de lazer, escolaridade, grupo social, fatos da infância, vivências da adolescência e interesses. Embora tenham sido encontradas peculiaridades, as temáticas se repetiam, atingindo o critério de saturação ${ }^{(19)}$ proposto pelo método empregado.

A coleta de dados ocorreu a partir da realização de duas sessões fonoaudiológicas individuais com seis pacientes com diagnóstico de $\mathrm{TGD}^{(3)}$. As sessões foram realizadas pela pesquisadora, privilegiaram a história de vida dos sujeitos e contaram com fotografias dos indivíduos e seus familiares. Cada sessão teve duração de 45 minutos e deixou-se o paciente livre para contar sua história a partir das fotografias expostas para ele. A pesquisadora direcionava o diálogo com questões sobre as situações vivenciadas, à medida em que o paciente ia contando os fatos.

Os seis sujeitos foram divididos em dois grupos, Grupo 1 e Grupo 2, sendo que os critérios de inclusão para participar do primeiro grupo foram: ter o diagnóstico de TGD do subtipo transtorno autista e estudar em escola especial. Para participar do segundo grupo, os critérios foram: ter o diagnóstico de TGD do subtipo transtorno de Asperger e estudar em escola regular.

As sessões dos sujeitos do Grupo 1 foram realizadas na escola especial onde estudam e as do Grupo 2 ocorreram em suas residências. Todas as sessões foram gravadas em vídeo e posteriormente transcritas para a seleção e análise dos episódios e direcionamento do conjunto dos dados para as categorias de análise.

\section{Resultados}

Quanto à caracterização dos sujeitos participantes desta pesquisa, os três pacientes do primeiro grupo estudam em uma escola especial voltada apenas para alunos com o diagnóstico de autismo e os três do segundo grupo estudam em escolas regulares. Dois desses sujeitos já frequentaram escola especial e, há sete anos, foram transferidos para escola regular, enquanto o outro sempre estudou em escola regular. Com relação às famílias, quatro (dois de cada grupo) moram com seus pais: um sujeito do primeiro grupo mora apenas com a mãe, que é divorciada do pai, e um do segundo grupo mora com a mãe, as irmãs e o atual marido da mãe. Um sujeito do primeiro grupo e dois do segundo possuem irmãos.

A partir dos dados obtidos nas duas sessões fonoaudiológicas com ênfase na história de vida dos pacientes, obtiveramse as seguintes categorias: lazer, escolaridade, rotina, grupo 
social, lembranças da infância, vivências da adolescência, interesses e religiosidade. Tais categorias não foram préestabelecidas, surgindo a partir dos processos dialógicos com os sujeitos e não foram entendidas como peculiaridades de temas abordados por sujeitos autistas, mas sim pertencentes a histórias de vida de qualquer sujeito. Tais resultados foram descritos nos Quadros 1 e 2.

\section{Discussão}

Todos os participantes da pesquisa relataram vivenciar atividades de lazer e demonstraram, em suas falas, prazer nessas situações. Considerando-se o desenvolvimento como o produto das experiências sociais, é possível dizer que tais vivências permitem ao indivíduo apropriar-se de fazeres e dizeres da cultura, sendo alavancas para sua aprendizagem e desenvolvimento ${ }^{(11)}$. É importante destacar que cinco sujeitos disseram ser acompanhados nos passeios pelos pais, tios, avós e, eventualmente, primos. Apenas um referiu realizar atividades de lazer com amigos dos pais, irmãs, cunhados e amigos. Essa situação reforça a dificuldade que os adolescentes autistas - assim como aqueles com alguma deficiência atrelada ao funcionamento - têm de crescer, assumir a postura adequada para a idade e participar de grupos com idades semelhantes às deles ${ }^{(20)}$. Tal observação não pode ser considerada típica do TGD, pois a adolescência é um período de mudanças tanto orgânicas quanto culturais ${ }^{(21)}$. Além disso, devido ao próprio modo de se comportar socialmente, advindo de dificuldades orgânicas e sociais, os adolescentes autistas não são facilmente incorporados aos grupos de sua faixa etária, restando-lhes, como opção fundamental, o contexto familiar. Dessa forma, cabe aos profissionais envolvidos orientar a família para que os sujeitos com TGD possam vivenciar, da forma mais adequada possível, as especificidades das diferentes fases etárias.

$\mathrm{Na}$ categoria escolaridade, observou-se que as situações que envolvem a escola são bastante significativas para os sujeitos, estando na lembrança de todos eles ao contarem suas histórias de vida. Cinco sujeitos apontaram para experiências positivas tanto nas escolas anteriores quanto na atual, e apenas um sujeito do Grupo 2 abordou pontos negativos de uma de suas escolas anteriores. A literatura mostra que a escola é um importante espaço de aprendizagem e desenvolvimento para os autistas, pois é o local onde eles têm a oportunidade de conviver com crianças normais e ter modelos, além de vivenciar situações ricamente permeadas pela linguagem e pela cultura ${ }^{(22)}$. Isso ficou claro quando os sujeitos contaram sobre festas, fantasias, olimpíadas e matérias escolares, trazendo em seus discursos aquilo que já vivenciaram. Apesar disso, existem diversas dificuldades no processo de inclusão dessas crianças, variando desde a falta de receptividade e qualificação dos professores até a incapacidade de estruturação do sistema educacional. Para minimizar tais complicações, é necessário que as crianças autistas sejam incluídas desde os primeiros anos de vida, quando inseridas na educação infantil ${ }^{(23)}$, sendo essa uma importante orientação do pediatra e do fonoaudiólogo.

Outro ponto a ser destacado nos dados são as lembranças negativas sobre a escola de um dos sujeitos do Grupo 2. Tal fato aparece na literatura ${ }^{(23)}$, mostrando que os autistas de alto funcionamento, incluindo transtorno de Asperger, são sensíveis às condições inadequadas da escola, interpretando tais sinais como uma rejeição, o que pode causar prejuízos nos anos decorrentes de escolaridade. No caso do sujeito da pesquisa isso não ocorreu, pois ele demonstra, com suas falas, estar feliz em sua escola atual. Porém, é importante que os profissionais que lidam com as crianças autistas em fase escolar fiquem atentos para a boa participação nos espaços escolares, orientando mudanças quando necessário.

Com relação ao grupo social, observou-se que todos os sujeitos fizeram referências às diversas pessoas de seus convívios sociais, envolvendo pais, irmãos, tios, primos, avós nas várias situações, tanto atuais quanto passadas. Esses relatos suscitam uma discussão paradoxal, uma vez que, ao mesmo tempo em que os autistas são entendidos como aqueles que apresentam alterações de relações sociais ${ }^{(24)}$, eles se mostram sensíveis ao outro, trazendo "seus outros" em seus discursos, demarcando a importância das pessoas em suas histórias de vida. É significativo abordar que nenhum dos sujeitos lembrou-se das pessoas que não eram da família e estavam presentes nas fotografias. Tal achado até poderia ser compreendido como mais um sintoma autístico; no entanto, o referencial histórico-cultural permite compreender que a memória é carregada de significações, sendo necessariamente atrelada às práticas sociais que vão se inscrevendo nos discursos. Isso ocorre na medida em que os sujeitos normais vão se apropriando da linguagem; porém, no caso das crianças autistas, as vivências precisam necessariamente ser interpretadas, vivenciadas e faladas por seus pais e familiares para que se tornem compreendidas, significativas e possam ser lembradas pelos sujeitos, e isso provavelmente não ocorreu, pois a situação passou e as lembranças se esvaíram, sem se tornarem objetos da fala e da emoção desses sujeitos autistas. 


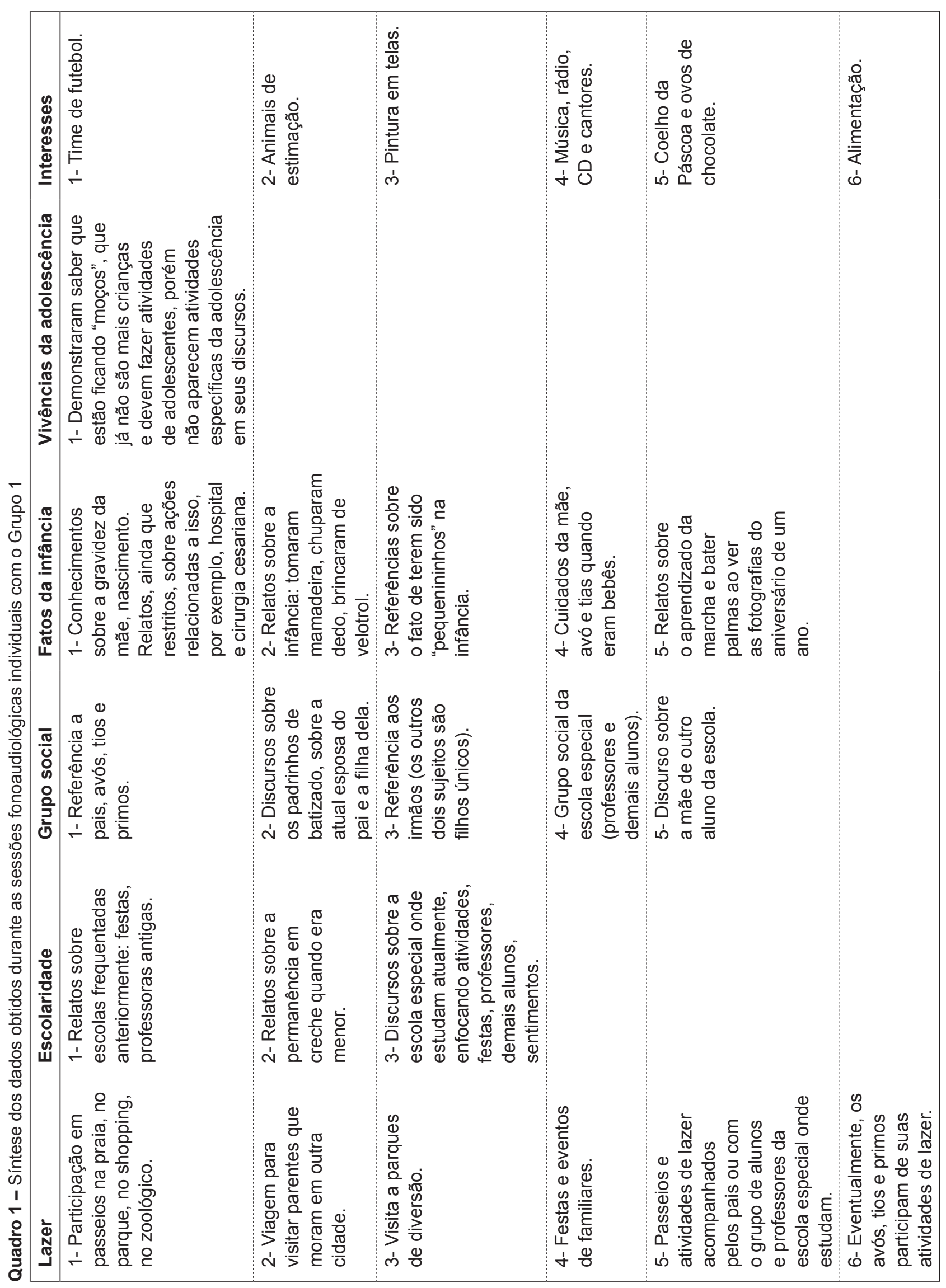




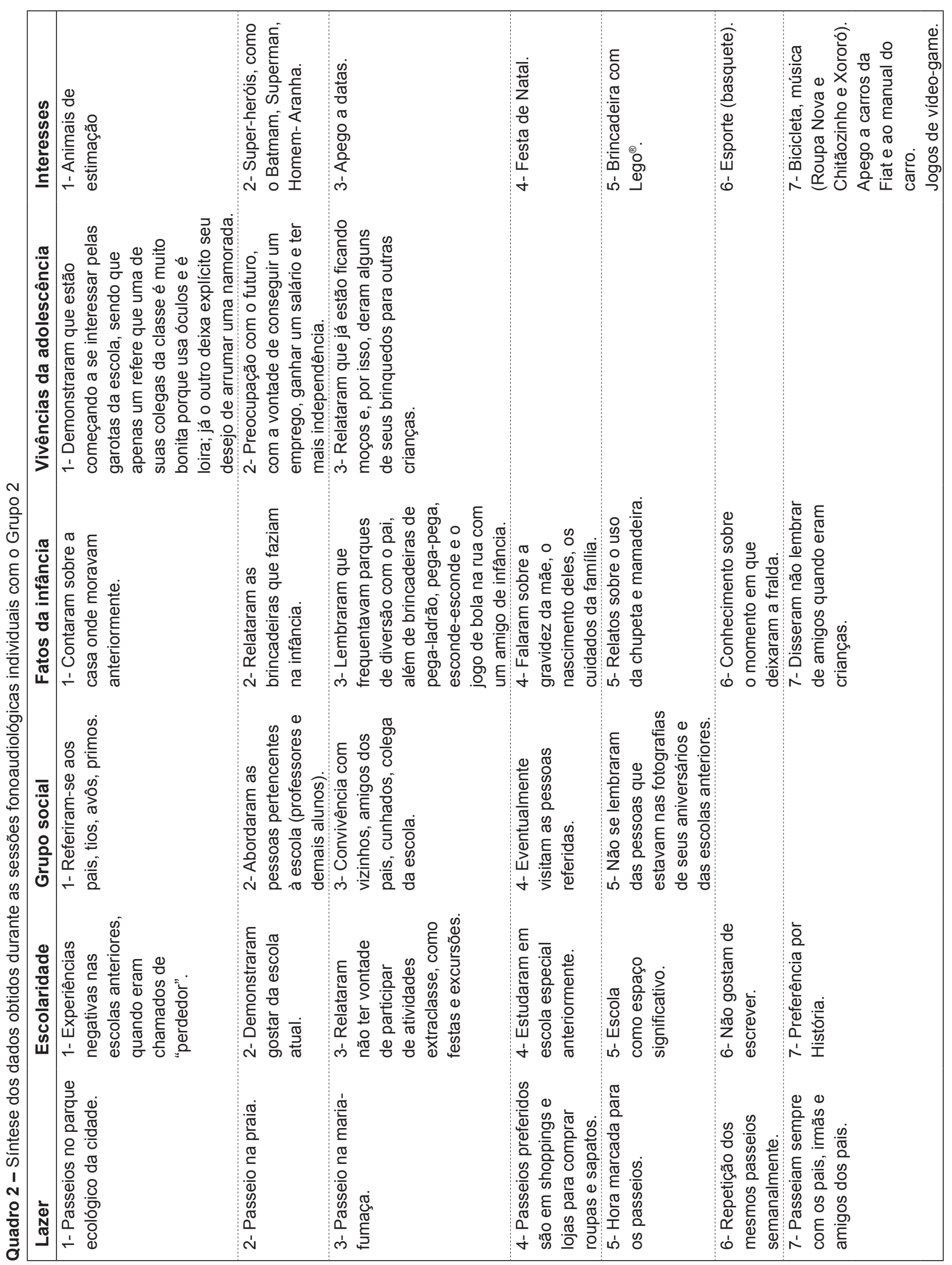


Na categoria "fatos da infância", foi possível notar que todos os participantes da pesquisa relatam saber sobre a gestação da mãe, o momento do nascimento, os hábitos de quando eram bebês, como chupeta, mamadeira, fralda, aprendizados, cuidados da família para com eles, diferenciação de tamanho entre a infância e o momento atual, por exemplo. Tais dados refletem que essas famílias, mesmo envolvidas com as questões próprias do autismo, valorizaram etapas importantes da vida dos filhos, oferecendo informações que compõem suas histórias de vida. Isso é fundamental para a sua constituição como sujeitos, pois a constituição humana vai correndo na medida em que o indivíduo passa a ser significado e a compor o discurso do outro ${ }^{(11)}$, além de possibilitar que o sujeito vá se apropriando da narrativa de sua história de vida e possa ir desenvolvendo o ato de narrar fatos, dando um salto em seu funcionamento linguístico-cognitivo ${ }^{(25)}$. Além disso, nessa categoria, alguns contaram que gostavam de brincar e citaram as brincadeiras de infância, e um dos sujeitos do Grupo 2 relembrou que tinha um amigo de infância com quem jogava bola.

Todos os dados desta categoria surgiram na medida em que a pesquisadora interagia com os sujeitos e no momento em que viram as fotografias, sendo a mediação importante para a retomada das lembranças, organização dos discursos e apropriação das histórias de vida. Isso ocorre, pois a memória do homem não é direta ou autônoma, mas sim dependente das situações sociais, da fala dos outros, das imagens, ou seja, é sempre mediada pelos signos ${ }^{(26)}$.

Em relação às vivências da adolescência, é importante ressaltar que os sujeitos do Grupo 1 demonstraram saber que já não são mais crianças, porém não fizeram referências às experiências específicas da idade. Já no Grupo 2, ficaram bastante evidentes as mudanças nas preocupações, sendo que dois sujeitos já demonstraram notar as diferenças entre eles e pessoas do sexo oposto. Um deles disse claramente nas entrevistas que, em breve, quer ter uma namorada. Além disso, um dos sujeitos disse se preocupar com o futuro, com o emprego e o autossustento. Sabe-se que a adolescência é um momento em a sociedade passa a atribuir ao sujeito um lugar diferente da infância, tendo por expectativa novos comportamentos, interesses e responsabilidades ${ }^{(21)}$. No caso dos autistas, as expectativas do grupo social são diferentes, atribuindo a eles sempre o lugar de crianças, comprometendo, assim, a formação da identidade de adolescente e, posteriormente, a de adulto ${ }^{(20)}$. Entretanto, os dados desta pesquisa mostraram que os sujeitos dos dois grupos estão no processo de passagem da infância para adolescência, uma vez que já sabem que cresceram (Grupo 1), já modificaram os interesses e preocupações (Grupo 2), mas ainda vivem de forma atrelada às significações da infância.

Tal situação paradoxal, entre a infância e a adolescência, ficou evidente também nos interesses dos sujeitos, quando eles relataram gostar tanto de atividades condizentes com a idade, como, por exemplo, futebol, música, pintura, esporte, vídeo game, quanto de objetos de interesses de crianças, como coelho da Páscoa e super-heróis. Apenas um dos cinco sujeitos expressou interesse excessivo e fixado em carros e manual de instrução de carros da Fiat; no entanto, esse mesmo paciente demonstrou interesse também em diversas outras atividades e objetos. Os dados encontrados nessa categoria contradizem um dos sintomas elencados nos parâmetros diagnósticos ${ }^{(3)}$, que é o de padrões restritos de interesse. Certamente, os dados desta pesquisa não indicam que o sintoma não ocorre, mas suscitam reflexões sobre o desenvolvimento social dos autistas, mostrando que os interesses não são eminentemente decorrentes de fatores orgânicos, mas sim incorporados a partir dos interesses do grupo social e da cultura circulante ${ }^{(11)}$.

O conjunto dos dados sinalizou também que o diagnóstico de TGD, tanto do subtipo autista quanto do Asperger, vem acompanhado não apenas dos sintomas já descritos pelos parâmetros diagnósticos, mas também de dificuldades na inserção social, inclusão escolar e de passagem pelas diferentes fases da vida (infância e adolescência). Em contrapartida, - e entendendo o desenvolvimento humano enquanto o produto das condições concretas de vida, das experiências vivenciadas e do modo como os sujeitos são significados pelo grupo social - as orientações familiares desde o diagnóstico passam a ser fundamentais para que os sujeitos autistas tenham um desenvolvimento satisfatório, vivam com independência e tenham melhor qualidade de vida.

Esta pesquisa permite considerar que se devem ofertar aos autistas ricas experiências sociais de lazer, de novas aprendizagens e inseri-los na escola desde a educação infantil, além de fornecer a eles dados sobre suas histórias de vida, possibilitar que as atividades cotidianas sejam condizentes com a faixa etária, incentivar relacionamento com diversas pessoas e permitir que eles exerçam atividades cotidianas de maneira autônoma. Além disso, pode-se concluir que as fotografias não são apenas um recurso terapêutico fonoaudiológico, funcionando como um signo mediador que possibilita o ato de lembrar, narrar ${ }^{(24)}$ e fornecer ao sujeito a condição de domínio do diálogo ${ }^{(27)}$, mas também podem ser importantes no contexto familiar, servindo à estruturação da história de vida do sujeito com TGD e, por consequência, à sua constituição enquanto ser humano. 
Sendo o pediatra o médico responsável pelos cuidados com a criança desde o nascimento até a adolescência - e com o qual a família estabelece uma relação de confiança -, cabe a ele auxiliar os pais e acompanhar o desenvolvimento dos pacientes, buscando estabelecer uma continuidade nas orientações e uma inter-relação com médicos psiquiatras, neurologistas e demais profissionais da saúde, tais como fonoaudiólogos, fisioterapeutas, terapeutas ocupacionais e

\section{Referências bibliográficas}

1. Assumpção Júnior FB, Kucznski E. Conceito e diagnóstico. In: Assumpção Júnior FB, Kucznski E. Autismo infantil: novas tendências e perspectivas. São Paulo: Atheneu; 2007. p. 1-17.

2. Tamanaha AC, Perissinoto J, Chiari BM. A brief historic review of the conceptions of Autism and Asperger syndrome. Rev Soc Bras Fonoaudiol 2008;13:296-9.

3. American Psychiatric Association. DSM-IV-TR: Manual diagnóstico e estatístico de transtornos mentais. 4a edição. Porto Alegre: Artmed; 2002. p. 99-103.

4. Sprovieri MH, Assumpção Jr FB. Dinâmica familiar de crianças autistas. Arq Neuropsiquiatr 2001;59:230-7.

5. Fávero MA, Santos MA. Autismo infantil e estresse familiar: uma revisão sistemática de literatura. Psicol Reflex Crit 2005;18:358-69.

6. Schmidt C, Dell'Aglio DD, Bosa CA. Estratégias de coping de mães de portadores de autismo: lidando com dificuldades e com a emoção. Psicol Reflex Crit 2007;20:124-31.

7. Fernandes FD. Families with autistic children: international literature. Rev Soc Bras Fonoaudiol 2009;14:427-32.

8. Barbosa MR, Fernandes FD. Qualidade de vida dos cuidadores de crianças com transtorno do espectro autístico. Rev Soc Bras Fonoaudiol 2009:14:482-6.

9. Coelho AC, lemma EP, Lopes-Herrera SA. Case report-deprivation of sensory stimuli and autistic behaviors. Rev Soc Bras Fonoaudiol 2008;13:75-81.

10. Góes MC. Relações entre desenvolvimento humano, deficiência e educação: contribuições da abordagem histórico-cultural. In: Oliveira MK, Souza DT, Rego TC. Psicologia, educação e as temáticas da vida contemporânea. São Paulo: Moderna; 2002. p. 95-114.

11. Pino A. As marcas do humano: as origens da constituição cultural da criança na perspectiva de Lev S. Vigotski. São Paulo: Cortez; 2005.

12. Pessoa JH. O exercício da pediatria nos dias atuais. Rev Paul Pediatr 2004;22:188-9 psicólogos. Assim, otimiza-se o funcionamento social, linguístico e cognitivo dos sujeitos autistas, proporcionando tranquilidade e conforto aos pais e familiares.

\section{Agradecimentos}

Agrademos à Coordenação de Aperfeiçoamento de Pessoal de Nível Superior (Capes) pelo apoio financeiro.

13. Delfrate CB, Santana AP, Massi GA. The acquisition of language in the child with Autism: a case study. Psicol Estud 2009;14:321-31.

14. Saad AG, Goldfeld M. A ecolalia no desenvolvimento da linguagem de pessoas autistas: uma revisão bibliográfica. Pró-Fono 2009;21:255-60.

15. Góes MC. A abordagem microgenética na matriz histórico-cultural: uma perspectiva para o estudo da constituição da subjetividade. Cad Cedes 2000;20:9-25.

16. Ginzburg C. Mitos, emblemas, sinais: morfologia e história. 2a ed. São Paulo: Companhia das Letras; 2003.

17. Vygotsky LS. Pensamento e linguagem. São Paulo: Martins Fontes, 2000.

18. Minayo MCS. O desafio do conhecimento: pesquisa qualitativa em saúde. São Paulo: Hucitec; 2004

19. Fontanella BJ, Ricas J, Turato ER. Saturation sampling in qualitative health research: theoretical contributions. Cad Saude Publica 2008;24:17-27.

20. Leite GA, Monteiro MI. A construção da identidade de sujeitos deficientes no grupo terapêutico-fonoaudiológico. Rev Bras Educ Espec 2008;14:189-200.

21. Bock AM. A perspectiva sócio-histórica de Leontiev e a crítica à naturalização da formação do ser humano: a adolescência em questão. Cad Cedes 2004;24:26-43.

22. Vygotsky LS. A formação social da mente. São Paulo: Martins Fontes; 2000

23. Camargo SP, Bosa CA. Competência social, inclusão escolar e autismo: revisão crítica da literatura. Psicologia \& Sociedade 2009;21:65-74.

24. Klin A. Autism and Asperger syndrome: an overview. Rev Bras Psiquiatr 2006;28(Suppl 1):S3-11

25. Perroni MC. Desenvolvimento do discurso narrativo. São Paulo: Martins Fontes; 1992.

26. Braga ES. O trabalho com a literatura: memórias e histórias. Cad Cedes 2000;20:84-102.

27. Coudry MI. Diário de narciso: discurso e afasia. 2a ed. São Paulo: Martins Fontes; 1996. 